\title{
A note on the heat kernel coefficients for nonminimal operators
}

\author{
B. Ananthanarayan \\ Centre for High Energy Physics, \\ Indian Institute of Science, Bangalore 560 012, India \\ e-mail: anant@cts.iisc.ernet.in
}

August 19, 2008

\begin{abstract}
We consider certain results for the heat kernel of nonminimal operators. The general expressions provided by Gusynin and Kornyak resulting from symbolic computation programmes for $n$ dimensions are evaluated for 4 dimensions which are checked against results given by Barvinsky and Vilkovisky. We also check that the results in flat space are consistent with earlier results of Guendelmen et al. We then consider a powerful construction of the Green function of a nonminimal operator by Shore for covariantly constantly gauge fields in flat spacetime, and employ dimensional arguments to produce a check on the gauge parameter dependence of a certain coefficient. The connection of the results for heat kernel coefficients emanating from the construction of Shore, to those from other techniques is hereby established for the first time.
\end{abstract}

Keywords: Heat-kernel, nonminimal operator, gauge fields, curved spacetime

PACS: 04.62.+v, 11.15.-q 


\section{Introduction}

A traditional approach to the evaluation of the divergent part of the generating functional of Green functions in field theory is the well-known heat kernel method, for a recent review see ref. 11. The coefficients in the asymptotic expansion of the 'diagonal' heat kernel elements are the well-known Seeley-DeWitt coefficients. These are typically obtained for so-called 'minimal' operators of the type $\left(-D^{\mu} D_{\mu}+X\right)$. Non-minimal operators typically involve bi-linears of the type $\left(-g_{\mu \nu} D^{\rho} D_{\rho}-(1 / \alpha-1) D_{\mu} D_{\nu}+X_{\mu \nu}\right)$, where the minimal case is obtained with $\alpha=1$.

In quantum field theory, the divergent part of the one-loop generating functional of Green functions may be expressed in terms of the second Seeley-DeWitt coefficient of certain differential operators. The subject is by now standard and is discussed in standard textbooks. Given a Lagrangian field theory, there is associated with this a differential operator denoted by $\mathcal{D}$ such that the one-loop generating function $\Gamma^{(1)}$ in the neighbourhood of $n=4$ is given in dimensional regularization by

$$
\frac{i}{2} \log \operatorname{det} \mathcal{D}=-\int d^{n} x \frac{1}{(n-4)} \operatorname{tr} E_{4}
$$

where $E_{4}$ is the second Seeley-DeWitt or heat kernel coefficient of the differential operator $\mathcal{D}$.

Typical differential operators that are considered are of the minimal kind. Non-minimal operators arise in gauge field theories in covariant gauges in general, where $\alpha$ is the gauge parameter. For the reasons mentioned above, the parameter is set equal to unity, which corresponds to the Feynman gauge1 1 . Differential operators also appear in curved spacetime which involve the Ricci tensor, curvature tensor and the scalar curvature. The traditional method of evaluating the corresponding heat kernel coefficients going under the name of the method of DeWitt does not work for the nonminimal case. Techniques used for such nonminimal operators go under the name of the method of Widom. There are techniques advanced in the literature which provide algorithms based on the method of Widom to compute the heat kernel coefficients, refs. 3, 4, 5. Note that nonminimal operators have also been considered in the context of noncommutative field theories 6 .

Results have been presented by Barvinsky and Vilkovisky (BV) in ref. [7] with curvature, where some of the results crosscheck those that were presented by in earlier literature. More recently, Pronin and Stepanyantz (PS) in ref. [8] have also considered the nonminimal case and find results consistent with those in ref. [7. The heat kernel coefficient corresponding to what is a surface term is not given by PS. Even more recently these operators have been studied by Gusynin and Kornyak (GK), in ref. 9] using symbolic computation and including the tensor denoted by $W_{i j}$ to account for gauge fields, and results have

\footnotetext{
${ }^{1}$ Note, for instance, that the divergent part of the one-loop generating functional in chiral perturbation theory with virtual pions has been computed only in the Feynman gauge [2].
} 
been provided for general case of $n$ dimensions. However, no attempt has been provided to compare the results from this to those of BV and PS, although it is a straightforward exercise. Here we provide such a comparison, as it is very important to check the results in every possible manner. Furthermore, it is important to look for an important crosscheck on the coefficient of the bilinears involving the gauge field strength tensor, and in particular of its dependence on the parameter $\alpha$. Results have been provided by Guendelman et al. [10, which are also based on symbolic computation methods.

In this regard, we show here that an elegant analytical approach is also available to accomplish this goal: we look at a completely different solution present in the literature which has not attracted attention to the best of our knowledge. This is the general case considered by Shore, ref. 11, for the case of a covariant gauge, but in flat spacetime. It is shown here that a study of his construction can provides a consistency check on the results obtained from the general expressions of GK for the case of four dimensions for the bilinear in gauge field strength. A different approach that also provides a proof of scuh gauge parameter independence is given by Avramidi [12.

In Sec. 2 we present an evaluation of the heat kernel coefficients for 4 dimensions from the general expressions given by of GK. We carefully compare the results given by BV and PS. It will turn out that one of the coefficients in our evaluation remains untested at this stage, but interestingly enough is independent of $\alpha$ for $n=4$. This coefficient along another combination of coefficients that is itself independent of $\alpha$ for $n=4$, which we will discuss, will be related to the work of Guendelman et al., who computed what is effectively this combination using symbolic computation. Yet other coefficients are listed here for the first time for $n=4$. We advance here an analytical argument in Sec. 3 where we consider in considerable detail the construction of Shore and work out the consequences for the heat kernel coefficients. In Sec. 4 we provide a discussion on the results and recapitulate the main results in this work.

\section{The Results of Gusynin and Kornyak for 4 dimensions}

A comprehensive treatment for the evaluation of the trace of the second SeeleyDeWitt coefficient termed $E_{4}$ is provided by GK 9 . In this paper, the trace of the Seeley-DeWitt coefficient evaluated explicitly in curved background, and in arbitrary gauge, and a list of $C_{i}, i=1, \ldots 14$, is provided in $n$ dimensions, in terms of a parameter $a$, where $a=1-1 / \alpha$. For all other definitions and conventions we refer to the paper of GK. Recall that the divergent part of the generating function is given by the spacetime integral of 


\begin{tabular}{|c||c||c||}
\hline \hline Term & Coefficient & Value \\
\hline \hline$R_{i j k l} R^{i j k l}-4 R_{i j} R^{i j}+R^{2}$ & $C_{9}$ & $-11 / 180$ \\
\hline$R_{i j} R^{i j}$ & $\left(4 C_{9}-C_{11}\right)$ & $\left(5 \gamma^{2}+10 \gamma-32\right) / 120$ \\
\hline$R^{2}$ & $\left(C_{13}-C_{9}\right)$ & $\left(5 \gamma^{2}+20 \gamma+28\right) / 240$ \\
\hline$R_{i j} X^{i j}$ & $-C_{10}$ & $-\gamma(\gamma+4) / 12$ \\
\hline$X_{i j} X^{i j}$ & $\left(C_{4} / 2+C_{5}\right)$ & $\left(\gamma^{2}+6 \gamma+12\right) / 24$ \\
\hline$R X_{i}^{i}$ & $-C_{14}$ & $-\left(\gamma^{2}+2 \gamma+4\right) / 24$ \\
\hline$X_{i}^{i} X_{j}^{j}$ & $C_{4} / 2$ & $\gamma^{2} / 48$ \\
\hline$W_{i j} W^{i j}$ & $C_{8}$ & $1 / 3$ \\
\hline \hline
\end{tabular}

Table 1: List of irreducible basis of tensor bilinears appearing in the divergent part of the one-loop generating functional, the corresponding coefficients, and their values.

$$
\begin{aligned}
& \operatorname{tr} E_{4}=(4 \pi)^{-n / 2} . \\
& {\left[-C_{1} D_{i} D^{i} X_{j}^{j}-C_{2} D_{i} D_{j} X^{i j}-C_{3} D_{i} D_{j} X^{j i}+\frac{C_{4}}{2}\left(X_{j}^{i} X_{j}^{j}+X_{i j} X^{i j}\right)+\right.} \\
& C_{5} X_{i j} X^{j i}+C_{6} X_{i j} W^{i j}-C_{7} W_{i j} X^{i j}+C_{8} W_{i j} W^{i j}+C_{9} R_{i j k l} R^{i j k l}- \\
& \left.C_{10} R_{i j} X^{i j}-C_{11} R_{i j} R^{i j}+C_{12} D_{i} D^{i} R+C_{13} R^{2}-C_{14} R X_{i}^{i}\right]
\end{aligned}
$$

We introduce a further parameter $\gamma \equiv a /(1-a)$ in order to have an effective comparison with the results of BV. We evaluate these for the case of 4 dimensions from the general formulae of GK and tabluate the (combinations) of coefficients in Tables 1 and 2. The results expressed in Table 1 are grouped to effect an easy comparison with known results in the literature. In particular, we are presenting those combinations of $C_{4}, C_{5}, C_{9}, C_{10}, C_{11}$ and $C_{13}$ which appear in the work of BV. The following may be noted:

(a) The sign convention for $C_{10}, C_{14}$ differs from that in BV.

(b) We regroup the terms to obtain the combination $\left(R_{i j k l} R^{i j k l}-4 R_{i j} R^{i j}+R^{2}\right)$ (surface term).

(c) Our results are in complete agreement with BV (also with those of PS, while noting that the latter omit the surface term). Note that in BV the divergent part of the generating functional involves $\left(\log L^{2}\right.$ ) (where $L$ is a large momentum scale) and it may be noted that one may map results obtained with cutoff regularization with those in dimensional regularization by identifying this with $-2 /(n-4)$.

(d) The last entry in Table 1 is not present in BV and needs to be verified independently, at least for the dependence on the gauge parameter. 


\begin{tabular}{|c||c||}
\hline \hline Coefficient & Value \\
\hline \hline$C_{1}$ & $\left\{\gamma\left(-6+9 \gamma+7 \gamma^{2}\right)+6\left(1-\gamma^{2}\right) \log (1+\gamma)\right\} /\left(36 \gamma^{2}\right)$ \\
\hline$C_{2}$ & $\left\{\gamma\left(96+150 \gamma+29 \gamma^{2}-6 \gamma^{3}\right)-6\left(16+33 \gamma+17 \gamma^{2}\right) \log (1+\gamma)\right\} /\left(72 \gamma^{2}\right)$ \\
\hline$C_{3}$ & $\left\{-\gamma\left(48+66 \gamma+19 \gamma^{2}-6 \gamma^{3}\right)+6\left(8+15 \gamma+7 \gamma^{2}\right) \log (1+\gamma)\right\} /\left(72 \gamma^{2}\right)$ \\
\hline$C_{6}$ & $-\left\{\gamma\left(288+756 \gamma+654 \gamma^{2}+156 \gamma^{3}-27 \gamma^{4}+4 \gamma^{5}\right)\right.$ \\
& $\left.-36(1+\gamma)^{2}(8+9 \gamma) \log (1+\gamma)\right\} /\left(288 \gamma^{2}(1+\gamma)\right.$ \\
\hline$C_{7}$ & $-\left\{\gamma\left(288+756 \gamma+510 \gamma^{2}+12 \gamma^{3}-27 \gamma^{4}+4 \gamma^{5}\right)\right.$ \\
& $\left.-36(1+\gamma)^{2}(8+9 \gamma) \log (1+\gamma)\right\} /\left(288 \gamma^{2}(1+\gamma)\right.$ \\
\hline$C_{12}$ & $\left\{\gamma\left(60+288 \gamma+95 \gamma^{2}\right)-30\left(2+9 \gamma+6 \gamma^{2}\right) \log (1+\gamma)\right\} /\left(360 \gamma^{2}\right)$ \\
\hline
\end{tabular}

Table 2: List of the remaining coefficients

(e) Despite the lack of details GK, one may try to compare the results in GK with those of Guendelman et al. [10]. In order to carry out a comparison with the results in the work of Guendelman et al, the following may be readily noted: with the identification $X_{i j}=-2 W_{i j}$, where $W_{i j}^{a b}=f^{a b c} F_{i j}^{c}$ (see eq. (2) in ref. [10]), the resulting coefficient of $W_{i j} W^{i j}$ is given by

$$
\left(2 C_{4}-4 C_{5}-2 C_{6}+2 C_{7}+C_{8}\right)=\frac{1}{12}\left(-25+n+\alpha^{n / 2-2}\right)
$$

which is in agreement with eq. (13) in ref. [10. Other terms in eq. (2) for this case in flat space vanish due to reasons of symmetry. The checks with the results of $\mathrm{BV}$ provide a check on the $\alpha$ independence of $\left(2 C_{4}-4 C_{5}-2 C_{6}+2 C_{7}\right)$, but that of $C_{8}$ can be checked only from the above. Thus, we show here for the first time the agreement of results obtained by two independent groups, which constitutes an important cross-check on the results.

(f) Despite all the cross-checks carried out so far, what is of interest to us here is to find an analytical argument for the feature of gauge independence of the combination on the left hand side of eq. (3) for $n=4$. In order to facilitate this latter, we will turn to the construction of Shore which is the subject of the next section.

In Table 2 we present the values obtained for those coefficients that do not appear in Table 1. These have not, to the best of our knowledge, appeared in the literature for 4 dimensions 2 These have a well-defined limit in the Feynman gauge $(\alpha=1, a=\gamma=0): C_{1}=1 / 6, C_{2}=C_{3}=C_{6}=C_{7}=0, C_{12}=$ $2 / 15$. These do not appear in $\mathrm{BV}$ as those accompanying $C_{1,2,3,12}$ vanish upon spacetime integration and those accompanying $C_{6,7}$ do not appear when gauge fields are not present.

\footnotetext{
${ }^{2}$ Expressed differently, this is a $\log \alpha$ dependence of some terms in the divergent part of the effective Lagrangian, which has been noted in the context of resonance saturation in chiral perturbation theory [13].
} 


\section{Heat-Kernel coefficients from Shore's construc- tion}

Shore considers the case of a covariantly constant field and obtains an explicit form for the entire heat kernel. In terms of the heat kernel obtained for the minimal case, an expression is provided for the nonminimal case as well. An expression for the one-loop divergence as the logarithm of the determinant of the relevant operator. For caveats regarding the use of the expressions in the $R$ gauge and conventional Lorentz gauge which will not affect our results, and for more details, we refer to the paper of Shore. A similar construction was also considered earlier by Endo [14.

There are several steps in the programme which is described below in some detail, keeping in mind that precise definitions may be found in the paper of Shore:

(a) The object of the study of Shore is the kernel $\mathcal{G}_{\mu \nu}^{a b}\left(x, y, ; t, m^{2}\right)$ for the vector operator $\mathcal{D}_{\mu \nu}^{a b}$ for arbitrary $\alpha$. It is defined by

$$
\begin{gathered}
\left(-D^{2} g_{\mu \lambda}+\left(1-\frac{1}{\alpha}\right) D_{\mu} D_{\lambda}+2 i g_{R} F_{\mu \lambda}+m^{2} g_{\mu \lambda}\right)^{a c} \mathcal{G}_{\lambda \nu}^{c b}\left(x, y, ; t ; m^{2}\right) \\
=-\frac{\partial \mathcal{G}_{\mu \nu}^{a b}\left(x, y, ; t ; m^{2}\right)}{\partial t}
\end{gathered}
$$

and satisfying the initial condition

$$
\mathcal{G}_{\mu \nu}^{a b}\left(x, y ; 0, m^{2}\right)=\delta_{a b} g_{\mu \nu} \delta(x, y)
$$

The covariant derivative $D_{\mu}^{a b} \equiv \partial_{\mu} \delta^{a b}-i g A_{\mu}^{c} t_{a b}^{c}$, where $A_{\mu}^{c}$ is the gauge field and $t_{a b}^{c}$ are the generators of the gauge group.

(b) If the condition $D_{\mu} m^{2}=0$ is satisfied then the kernel for nonzero mass factorizes into

$$
\mathcal{G}_{\mu \nu}\left(x, y ; t ; m^{2}\right)=\mathcal{G}_{\mu \nu}(x, y ; t ; 0) \exp \left(-m^{2}(y) t\right)
$$

(c) There is an ansatz that relates the solution for the nonminimal operator to that of the minimal operator $(\alpha=1)$ with $m^{2}=0$, for the case of covariantly constant fields. The heat kernel for the minimal operator with $m^{2}=0$ is denoted by $\overline{\mathcal{G}}_{\mu \nu}(x, y ; t)$, and the corresponding Green's function is denoted by $\bar{G}(x, y)$. Armed with this, the function $\mathcal{H}_{\mu \nu}$ is constructed and the desired heat kernel for the zero mass case is constructed via

$$
\mathcal{G}_{\mu \nu}(x, y ; t, 0)=\overline{\mathcal{G}}(x, y ; t)+D_{\mu} D_{\lambda}\left\{\mathcal{H}_{\lambda \nu}(x, y ; t)-\mathcal{H}_{\lambda \nu}(x, y ; t / \alpha)\right\} .
$$

(d) This expression has an remarkable property in that the $\alpha$ dependence factors out completely. An explicit expression for the heat kernel of the minimal operator with $m^{2}=0$ for the case of the covariantly constant gauge field strength 
is provided, and eventually an expression for the logarithm of the determinant of the operator.

(e) Consider now the result presented in eq.(4.67) in ref. [11. In this expression, for our purposes it suffices to suppress the trace over the gauge indices (Tr), and instead introduce a constant $C$, and inserting the spacetime trace (tr) for the case of $n=4$, we write down the schematic expression for the logarithm of the determinant of the differential operator as:

$$
\begin{gathered}
\frac{1}{C} \log \operatorname{det} \mathcal{D}=-(4 \pi)^{-n / 2} \int d^{n} x \\
\int_{0}^{\infty} d t t^{-1-n / 2}\left[(g F t)^{2} / \sin ^{2}(g F t)\left\{(4 \cos (2 g F t)-1) e^{-m^{2} t}+e^{-\alpha m^{2} t}\right\}\right]
\end{gathered}
$$

The divergent part is now obtained by expanding out the parts of the integrand that do not involve the exponentials in powers of $t$. Recalling that

$$
\begin{gathered}
\int_{0}^{\infty} d t t^{r-1-n / 2} e^{-m^{2} t}=\Gamma(r-n / 2)\left(m^{2}\right)^{n / 2-r} \\
\Gamma(-k+\epsilon)=\frac{(-1)^{k}}{k !}\left(\frac{1}{\epsilon}+\ldots\right), k=0,1,2, \ldots
\end{gathered}
$$

we can readily see that divergent part in the spacetime integrand now reads for the case of four dimensions:

$$
-\frac{1}{2} \frac{1}{16 \pi^{2}} \frac{1}{n-4}\left(3+\alpha^{2}\right) m^{4}+\frac{1}{3} \frac{g^{2}}{16 \pi^{2}} \frac{1}{n-4} 20 F^{2} .
$$

It may be recalled here that the residue of the pole at 4 in the spacetime integrand of $(-1 / 2) \log \operatorname{det} \mathcal{D}$ is the trace of the 2nd Seeley-DeWitt coefficient, keeping in mind that Shore employs the Euclidean generating functional.

(f) What is of note above is that the $F^{2}$ piece is independent of $\alpha$ which may be inferred from dimensional considerations.

To summarize, what we obtain from the analysis of the construction of Shore is the prediction that the $m^{4}$ piece in the divergent part is proportional to $\left(3+\alpha^{2}\right)$, and that the $F^{2}$ piece is independent of $\alpha$.

In order to make contact with the results of the previous section, it may be readily checked that, up to the factor $C$,

(A) for the case of $X_{i j}=m^{2} g_{i j}$ we get back the $\left(3+\alpha^{2}\right)$ dependence for the coefficient for $m^{4}$ by evaluating $\left[16\left(C_{4} / 2+C_{5}\right)+2 C_{4}\right]$ from Table 1 ,

(B) we find a simple justification for the $\alpha$ independence of the combination given in eq. (3). 


\section{Discussion and summary}

We have considered in some detail the implications of the results given in the work of GK for four dimensions. The results check those of BV, including the one result in the latter that was not checked earlier by the results of PS, namely that of the surface contribution. In addition, we have considered the remarkable construction of Shore for the case of covariantly constant fields, for which a complete construction of the Green function for the nonminimal case is provided and employ this to obtain the heat kernel coefficients for a simplified representation. The one corresponding to the $m^{4}$ term is shown to have a $\left(3+\alpha^{2}\right)$ dependence which agrees with the results of GK and that of BV and PS. In addition the construction of Shore provides a simple dimensional argument for why the $F^{2}$ term should be independent of $\alpha$. This agrees with the observation of Guendelman et al. which was found using symbolic computation. The remaining coefficients $C_{1,2,3,6,7,12}$ are also evaluated in 4 dimensions.

While it would be interesting to demonstrate that the construction of Shore is indeed consistent with the method of Widom in a formal manner, we have demonstrated instead that the results from this construction are in agreement with the heat kernel coefficients obtained from the Widom method.

\section{Acknowledgements:}

We thank the Department of Science and Technology, Government of India for support during the course of this work. Special thanks are due to H. Leutwyler for discussions. Discussions with B. Moussallam, S. Nampuri, A. K. Nayak, S. Mallik, J. Samuel, K. Shivaraj, A. Upadhyay and S. Vaidya are acknowledged. We thank Prof. M. Shaposhnikov at the Ecole Polytechnique Fédérale de Lausanne for his hospitality when part of this work was done, and the Indo-Swiss Bilateral Research Initiative for support. 


\section{References}

[1] D. V. Vassilevich, Phys. Rept. 388, 279 (2003) arXiv:hep-th/0306138.

[2] R. Urech, Nucl. Phys. B 433, 234 (1995) arXiv:hep-ph/9405341,

H. Neufeld and H. Rupertsberger, Z. Phys. C 71, 131 (1996) arXiv:hep-ph/9506448.

See also M. Knecht and R. Urech, Nucl. Phys. B 519, 329 (1998) arXiv:hep-ph/9709348].

[3] V. P. Gusynin, Phys. Lett. B 225, 233 (1989).

[4] V. P. Gusynin and E. V. Gorbar, Phys. Lett. B 270, 29 (1991).

[5] V. P. Gusynin, E. V. Gorbar and V. V. Romankov, Nucl. Phys. B 362, 449 (1991).

[6] A. Strelchenko, Int. J. Mod. Phys. A 22, 181 (2007) arXiv:hep-th/0608134.

[7] A. O. Barvinsky and G. A. Vilkovisky, Phys. Rept. 119, 1 (1985).

[8] P. I. Pronin and K. V. Stepanyantz, Nucl. Phys. B 485, 517 (1997) arXiv:hep-th/9605206.

[9] V. P. Gusynin and V. V. Kornyak, Nucl. Instrum. Meth. A 389, 365 (1997).

[10] E. I. Guendelman, A. Leonidov, V. Nechitailo and D. A. Owen, Phys. Lett. B 324, 160 (1994) arXiv:hep-th/9312138.

[11] G. M. Shore, Annals Phys. 137, 262 (1981).

[12] I. G. Avramidi, J. Math. Phys. 36, 1557 (1995) arXiv:gr-qc/9403035.

[13] B. Moussallam, Nucl. Phys. B 504, 381 (1997) arXiv:hep-ph/9701400.

[14] R. Endo, Prog. Theor. Phys. 71, 1366 (1984). 